\title{
Antennas Performance Comparison of Multi-Bands for Optimal Outdoor and Indoor Environments Wireless Coverage
}

\author{
Karrar Shakir Muttair ${ }^{1}$, Ali Z. Ghazi Zahid ${ }^{2}$, Oras A. Shareef Al-Ani ${ }^{3}$, Ahmed Mohammed Q. AL- \\ Asadi $^{4}$, Mahmood F. Mosleh ${ }^{5}$ \\ ${ }^{1,2}$ Department of Computer Techniques Engineering, College of Technical Engineering, The Islamic University, Najaf, \\ Iraq. \\ 3,5 Department of Computer Engineering Techniques, Electrical Engineering Technical College, Middle Technical \\ University, Baghdad, Iraq. \\ ${ }^{4}$ Al-Najaf Al-Ashraf International Airport, Najaf, Iraq.
}

\section{Article Info \\ Article history: \\ Received Jun. 12, 2021 \\ Revised Sep 26, 2021 \\ Accepted Oct 17, 2021}

\section{Keywords:}

RSS

Coverage Ratio

Path Attenuation

Path Gain

Optimal Wireless Coverage

RSQR

Antennas Performance

\begin{abstract}
This paper aims to implement a wireless Wi-Fi network (Indoor and Outdoor) in order to cover the environment of the Oxford Institute (to learn languages and computer skills) in the best methods and lowest cost in order to provide Wi-Fi service for faculty members and all members of the administrative board and students. The realistic three-floor indoor and outdoor environments of the Institute were designed with Wireless InSite Package (WIP). In addition, emphasis was focus on the use of two types of transmitting devices (Directional and Omni-Directional). The aim of using these two devices is to determine which device is better to cover the Institute's environment well. In this work, a different frequency bands scenario was used to determine which band is suitable for coverage and stability of the wireless network. These bands are $\mathrm{S}$-Band $(2.4 \mathrm{GHz}), \mathrm{C}-\mathrm{Band}(5 \mathrm{GHz}), \mathrm{C}-\mathrm{Band}(10 \mathrm{GHz}), \mathrm{Ku}-\mathrm{Band}$ $(15 \mathrm{GHz}), \mathrm{Ka}-\mathrm{Band}(28 \mathrm{GHz})$, and MmWave (39GHz). Moreover, the focus has been on the most important basic parameters to determine the performance level of the two devices (Directional and Omni-Directional) as well as to determine the performance level of the wireless network. The most important of these parameters are Path Losses $\left(L_{P a t h}\right)$, Path Gain $\left(G_{P a t h}\right)$, Received Signal Strength (RSS), Strongest Received Power, Coverage Ratio (CR), and Received Signal Quality Ratio (RSQR). According to the results that emerged, it was observed that Omni-Directional antennas are much better than Directional antennas, especially in NLOS (None-Line-of-Sight) regions. It was also noted that $\mathrm{CR}, L_{P a t h}$, and RSS at $\mathrm{S}-\mathrm{Band}(2.4 \mathrm{GHz})$ are much better than the rest of the bands, so that the CR and the RSQR at this band reach $83.2184 \%$ and $95.7383 \%$, respectively. While at the MmWave-Band $(39 \mathrm{GHz})$, it reaches $31.0345 \%$ and $70.7937 \%$ respectively.
\end{abstract}

Copyright () 2021 Institute of Advanced Engineering and Science. All rights reserved.

\section{Corresponding Author:}

Karrar Shakir Muttair,

Department of Computer Techniques Engineering,

College of Technical Engineering,

The Islamic University, Najaf, Iraq.

Email: karraralnomani123@gmail.com, karrar.alnomani@iunajaf.edu.iq

\section{INTRODUCTION}

In recent years, wireless communication technology has developed rapidly and has brought many updates and challenges in the design of wireless devices, as well as the introduction of high-performance antennas that help improve communication between users in various environments, whether indoor, outdoor or mixed [1]. In addition, the design of the wireless communication networks has become more developed, complexity and safe in various methods about how to effectively manage the network which is increasingly driven attention for the development of the network management systems [2]. Just like in establishing a 
network, it should be accurately designed the network layout before implementing it, this is to make sure that you are establishing any network that has good security since security is considered the main concern in designing a network [3].

Nowadays, antennas that operate on multiple and wide frequency bands are the most used in advanced communication networks, because these antennas can be used in various advanced standards of the Global Systems for Mobile Telecommunications (GSM), the Universal Mobile Telecommunications Systems (UMTS), Long-Term-Evolution (LTE), Wireless Fidelity (Wi-Fi) in mobile communication systems and various other devices [4, 5, 6]. Furthermore, most indoor and outdoor wireless communication networks contain nodes that are equipped with Omni-Directional antennas. Because the patterns of these antennas are transmitting signals in all directions, all nodes adjacent to other nodes are prevented from transmitting to avoid collisions between nodes $[7,8]$. Therefore, the capacity of wireless communication networks that contain an omnidirectional antenna is limited in use due to the continuous and high interference, so these networks using Omni-Directional antennas are called Wireless Omni-Directional Networks (WONs) [9].

On the other hand, other studies focus on the use of Directional antennas instead of Omni-Directional antennas in outdoor networks. When comparing directional antennas with multi-directional antennas, the transmission patterns of directional antennas are that data are directed in one specific direction [10]. Thus, the use of directional antennas in external networks allows sending more simultaneous transmissions in the pair vicinity of communication nodes, and the use of these antennas will reduce the interference between nodes close to each other [10]. The networks that use these antennas are called Directional Wireless Networks (DAWNs), which have a wide capacity and high than WONs because directional antennas use to transmit data over longer distances, so they are suitable in external wireless communication networks [11].

In recent research, emphasis has been placed on designing Wi-Fi for outdoor environments. These environments were designed with the use of the WIP. The main objective of this design and work is to determine the effects that affect the path of signals when they are transmitted from the transmitting to the receiving antennas. According to the results, the researchers indicated that Wi-Fi tends to lead to high successive losses and attenuation when the transmitting device is located between obstacles and barriers [12]. In another study, the focus was on designing a simulation scene model for a campus consisting of several floors using WIP. This work focuses on the frequencies are 0.85 and $1.9 \mathrm{GHz}$, to determine which is the best for campus coverage. Therefore, the transmitters were installed on the outside campus building, and the receiver devices were placed on each floor inside the campus building. The main purpose behind this design was to cover the campus in a good method as well as the effects that affect the paths as they move from the outside building to the interior. After making comparisons between the two frequencies, the researchers indicated that the path that carries the frequency of $0.85 \mathrm{GHz}$ is better and has less attenuation and noise than the frequency of $1.9 \mathrm{GHz}$ [13]. Moreover, in another recent study, the simulated environment scene was designed using WIP. The purpose of this design was to measure the performance of antennas (Directional and Omni-Directional) to study the path propagation and which antenna is better to cover the target environment. According to the results explained by the researchers and emerging from the WIP, the results proved that the Directional antenna is better than the Omni-Directional antenna, especially in NLOS environments that contain barriers because the directional antenna directs the beam in one direction. While the results also confirmed that the OmniDirectional antenna is better in the LOS (Line-of-Sight) regions than the directional antenna [14].

In this paper, a wireless network will be designed for different (indoor and outdoor) environments. The aim of designing this network is to obtain good coverage for faculty members and all students of the Oxford Institute (for learning languages and computer skills). In this work, two devices will focus on transmitting signals, the first device is a directional antenna transmitting and the second device is an Omnidirectional antenna transmitting. The main aim of using these two devices is to know which is better to cover the Institute's environment. In addition, different frequency bands were used in the scenarios of this work, namely S-Band (2.4GHz), C-Band (5GHz), C-Band (10GHz), Ku-Band (15GHz), Ka-Band (28GHz), and MmWave (39GHz). The main purpose of using different bands is to determine which from these bands are best suited to coverage the Institute's internal and external environment.

The rest of the sections for this paper are arranged as follows: Section 2 will present the most important parameters for measuring the performance of the antennas used in this paper. Section 3 will present the main and necessary parameters to determine the performance of the wireless network designed in this paper. Section 4 will provide a detailed explanation of the classification for the spectral frequency bands. Section 5 will present the indoor and outdoor designs for the target environment, as well as present all the scenarios related to the characteristics of the transmitter and receiver devices, as well as the used spectral frequencies that have been focused on in this paper. In Section 6, all scenarios for the results will be presented with an analysis and discussion of these results in detail. Finally, conclusions for the proposed work in this paper and suggestions for future work will be presented in Section 7. 


\section{ANTENAS PERFORMANCE MEASUREMENT PARAMETERS}

\subsection{Path Attenuation or Path Loss $\left(L_{P a t h}\right)$}

It is a decrease in the power density of electromagnetic waves as they propagate into space. It is considered an essential and major component in analyzing the performance of various communication network systems [15]. It is commonly used in various wireless communications. The attenuation of the path is the result of many influences and obstacles, the most important which are environmental conditions (for example, rural or urban), as well as affected by the medium of diffusion, whether air (dry, cold, hot, or humid) and also affected by obstacles such as reflections, absorption, materials that disperse the signal paths, deflections, refractions, and other influences. In addition, the distance between the transmitting and receiving antennas has a great effect on path attenuation and losses. It can be calculated based on Eq. (1) [16].

$$
L_{P a t h(d B)}=-10 \log _{10}\left(\frac{\lambda^{2} G_{T} G_{R}}{(4 \pi)^{2} * R^{2}}\right)+G_{T(M a x)(d B i)}+G_{R(M a x)(d B i)}+L_{S(d B)}
$$

Where $\lambda$ is the path wavelength, $R$ is the distance between the transmitter antenna and the receiver antenna, $G_{T}$ is the transmit antenna gain in the receiver direction, $G_{R}$ is the receiving antenna gain in the transmitter direction, $L_{S(d B)}$ is the additional losses incurred in communication systems in (dB), $G_{T(M a x)(d B i)}$, and $\mathrm{G}_{\mathrm{R}(\mathrm{Max})(\mathrm{dBi})}$ are the max gains of the transmitter and receiver antennas in $(\mathrm{dBi})$, respectively.

\subsection{Path Gain $\left(G_{\text {Path }}\right)$}

The path gain is one of the basic measurements and is equivalent to the path attenuation, but it is the inverse of the value. It can be used in place of path losses to measure the performance of path propagation in various environments because it is equivalent to the measurement of attenuation. It is sometimes used instead of a path loss measure when it provides a more appropriate scenario or method for presenting results. In addition, it is affected by many influences that make it gradually decrease. These effects are the same as those that affected the measurement of attenuation. It can be calculated through Eq. (2) [17].

$$
G_{P a t h(d B)}=-L_{P a t h(d B)}
$$

\subsection{Received Signal Strength (RSS)}

It is sometimes called the Received Signal Strength Index (RSSI) and measures the received power of the received signals [17]. RSS values are measured in $\mathrm{dBm}$, and the measured values range from $>$ $-50 \mathrm{dBm}$ (very excellent signal) to less than $-70 \mathrm{dBm}$ (very weak signal) as shown in Figure 1.

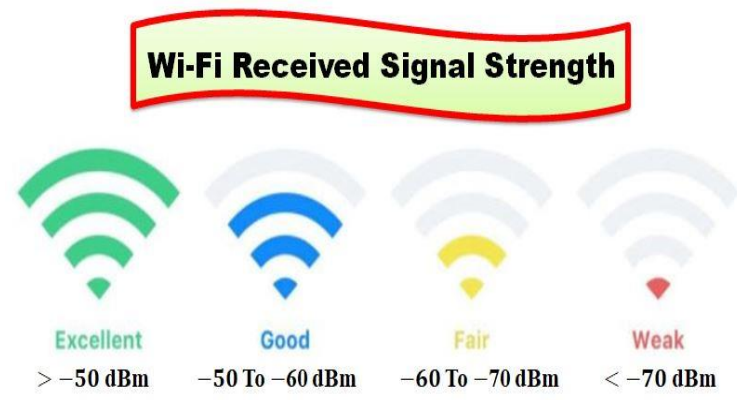

Figure 1. The values of Wi-Fi power strength that reaching users subscribed to the wireless network [18].

There are several factors that negatively affect the low power of the received signal, and the most important these factors are:

1- The distance between the transmitter antenna and the antennas of the deployed received points in the simulation environment.

2- The materials used in the construction of walls and barriers play a role by scattering, reflection, absorption, or diffraction of the received signal path.

3- The materials used to manufacture things inside or outside the environment. 
4- The number of the human, as the human body is prevented from penetrating the transmitted signals and some other signals are dispersed due to the clothes that humans wear. For this, it can be calculated according to Eq. (3) [17].

$$
\operatorname{RSS}_{(d B m)}=\left(\frac{\lambda^{2} \beta P_{T}}{(4 \pi R)^{2}}\right)+I_{T}+N_{T}
$$

Where $P_{T}$ is the rate of the transmitted power from the transmitter, $I_{T}$ is the total of the interference between paths, $N_{T}$ is the total of the noise.

\section{ANTENNA APPROPRIATE ESTIMATE PARAMETERS FOR ENVIRONMENT COVERAGE}

To measure the performance of the antennas in order to obtain the appropriate coverage and which antenna is better (Omni-Directional or Directional) to the environment coverage from outdoor to indoor. The most important parameters that have been focused on to determine or know the stability of the wireless network are the Received Signal Quality Ratio (RSQR), Coverage Ratio (CR).

\subsection{Received Signal Quality Ratio (RSQR)}

It is a necessary measure in order to determine the extent of the losses that accompany paths when transmitted through space from the transmitting antennas to the receiving points antennas. It can be calculated based on the power strength that reaches for each receiving point, and as shown in Eq. (4) [18].

$$
\operatorname{RSQR}_{(\%)}=\frac{\sum_{i=1}^{T_{R p}} 2 *\left(P_{R(i)}+100\right)}{T_{R p}} \%
$$

Where $T_{R p}$ is the total number of received points deployed in the simulation environment.

\subsection{Coverage Ratio (CR)}

Measuring and calculating the coverage performance of the target environment is very necessary, especially in wireless communication networks. Through the value that comes from the measurements, we will determine which antenna (Directional or Omni-Directional) is most appropriate to cover the simulation environment. It can be calculated based on the points that receive excellent signals, as shown in Eq. (5) [15].

$$
C R_{(\%)}=\frac{T M_{R p}}{T_{R p}} * 100
$$

Where $T M_{R p}$ is the total number of the maximum power (excellent power) that reached the receiving points deployed in the target environment.

\section{SPECTRUM FREQUENCY BANDS CLASSIFICATION}

In general, the frequencies classify the spectral bands into five classifications, the first band is called the S-band, the second band is called the C-band, the third band is called the Ku-band, the fourth band is called the Ka-band, and these four classifications can be called the Microwave band, while the fifth band is called the Millimeter Wave (MmWave). These classifications will be detailed explained in each subsection.

\subsection{S-Band Spectrum Frequency}

The S-band is an electromagnetic spectrum range between frequencies ( 2 to $4 \mathrm{GHz}$ ) as shown in Figure 2. This band is named according to the standards by the Academic Institute (Institute of Electrical \& Electronics Engineers (IEEE)). It goes beyond the traditional levels between the Ultra high-frequency (UHF) and Super high-frequency (SHF) bands at $3 \mathrm{GHz}$ [15]. This band is used in very wide fields such as airport monitoring to control the level of air movements. It is also used in weather radar systems and ship radar systems as well as satellite communications and international space tracking stations by NASA to communicate around space [19]. In addition, the S-band also contains an (Institute for Supply Management (ISM)) frequency of $2.4 \mathrm{GHz}$ which is used in a wide range of applications and in most fields. The Microwaves that need low energy and the most important of these Microwaves that use a $2.4 \mathrm{GHz}$ ISM license such are wireless smartphones, wireless headphones (Bluetooth), wireless communication networks (Wi-Fi), smart car locks, as well as remote baby monitors, and various devices that used in the medical fields [19]. 


\subsection{C-Band Spectrum Frequency}

The C-band is the Microwave band which ranges between $(4-12 \mathrm{GHz})$ as shown in Figure 2. This band has been named by an Academic Institute, the Institute of Electrical and Electronics Engineers (IEEE), as it is part of the electromagnetic wave spectrum [15]. In addition, this band is used in many fields and applications, the most important of which are satellite-based communications, as well as various applications of wireless direct Wi-Fi networks and wireless smartphone devices. Furthermore, this band is used in most surveillance, radars, and weather systems [19].

\subsection{Ku-Band Spectrum Frequency}

It is the frequency spectrum, which ranges from $(12$ to $18 \mathrm{GHz})$ as shown in Figure 2. This band is widely used in wireless communications. This band is characterized by a high capacity with flexibility, economical and better productivity than $\mathrm{C}$-Band, which makes this band suitable for all applications, the most important of which are satellite communications, weather forecasting, astronomy, navigation, satellite phone, satellites, military satellites, satellite radio, the internet via satellite, and others [20].

\subsection{Ka-Band Spectrum Frequency}

This frequency spectrum is considered more productive in transmitting the largest amount of data compared to the $\mathrm{Ku}$-band spectrum so that the spectrum band of this band ranges between $(18-30 \mathrm{GHz})$ as shown in Figure 2. In addition, it has other uses in the fields of military systems and Microwave devices with high-capacity features that require high wavelength and wide transmission of data, as well as it has many uses in radar systems, espionage, defense systems, and others [21].

\subsection{MmWave Band Spectrum Frequency}

It is also known as MmWave, the spectrum band of this wave is used in various and many highprecision and speed applications, as well as in advanced communications applications that require a high spectrum in order to transmit the largest amount of data to users, as well as national security, defense applications, and others [22]. The International Telecommunication Union (ITU) is the one who defined this spectrum with different and very high frequency (VHF), where the frequencies for this wave range from (30 to 300) $\mathrm{GHz}$ as shown in Figure 2. One of the most important characteristics through the use of the MmWave is the transfer of very large data amounts. The increase in the amount of data transmitted requires the use of high frequencies [22]. This is a working principle that comes in MmWaves, where high frequencies are used to transmit large amounts of information at the highest speed, such as data transmitted through many Television (TV) channels, computer data, and others [23].

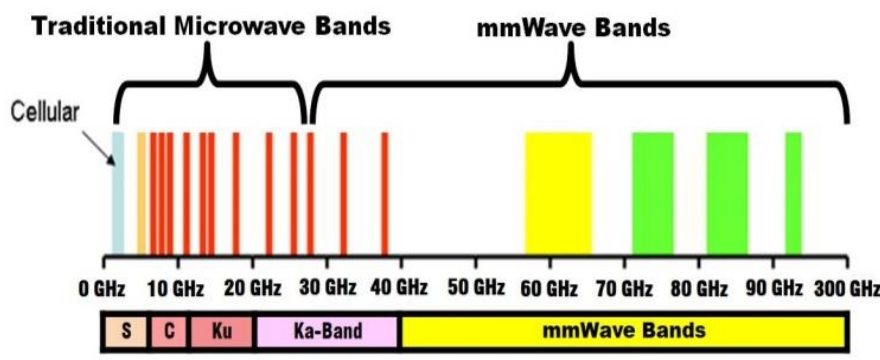

Figure 2. Classification of bands for the various frequency spectrum [19].

\section{PROPOSED CASE NETWORK STUDY MODEL}

In this work, emphasis is placed on the case study of the Oxford Institute (for Languages and Computer Skills Learning) located in Baghdad. This focus was necessary to cover the institute's environment with a Wi-Fi network to the best methods and provide a good network for the faculty members and all the institute's corridors. The institute mainly consists of two regions, the first region is the indoor region that consists of three floors where the ground floor contains rooms for the director of the institute and his assistants, as well as the administrative units' rooms and faculty rooms, while the first, second and third floors contain classrooms, offices, observation rooms, as well as health clinics. While the second region is the outdoor region and it consists of student rest areas, gardens, and parking spaces. A case study model for the institute's environment that was built using the WIP is shown in Figure 3. The WIP is one of the best software to simulate realistic networks, whether internal, external, or mixed.

The materials used in construction and the thickness of each material are listed in Table 1. The brick material is used to build the internal and external walls of the institute's environment, and the concrete material is used to build the floor and ceiling of the institute's environment. While metal was used in the design of 
exterior doors and window frames. Dense Foliage is used to construct gardens and various student rest regions. In addition, wood material is used to design the interior doors of every classroom. Finally, glass is used as packaging for interior and exterior door and window openings.

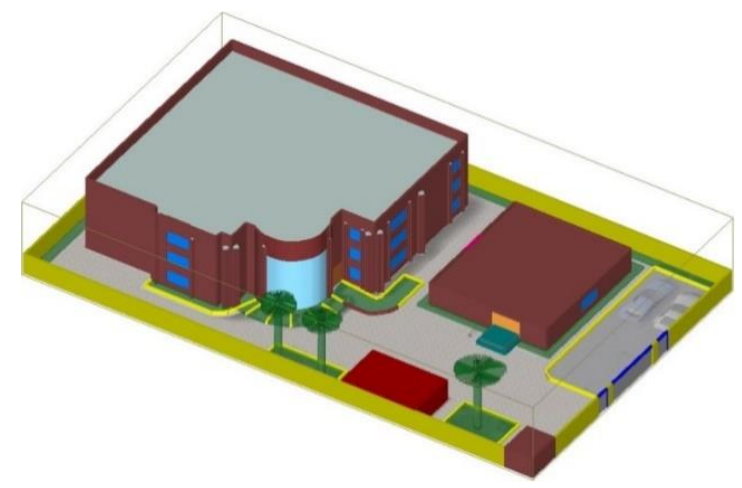

Figure 3. A case study model for the institute's environment (Indoor and Outdoor regions) was built using the WIP.

Table 1. Types and thickness of materials used in building the environment of the simulation institute.

\begin{tabular}{cc}
\hline Types of Materials & Thickness $(\mathbf{m})$ \\
\hline Brick & 0.28 \\
Concrete & 0.3 \\
Metal & 0.0625 \\
Dense Foliage & 0.02 \\
Wood & 0.045 \\
Glass & 0.03 \\
\hline
\end{tabular}

All materials used in building any simulated environment in the WIP have an electrical analog characteristic which is represented by conductivity and permittivity, and according to ITU's organization which is responsible for the effect of frequencies and materials on the direction of the signal path, it was assumed that the conductivity changes with the change of frequency and also changes with the change of materials used in building the simulation environment for some materials such as (concrete, wood, and glass), while the permittivity value is not changed when the frequency changes and the material changes [18]. The conductivity $(\sigma)$ and permittivity $(\varepsilon)$ are calculated on the basis of ITU for all frequencies according to Eq. (6) and Eq. (7). Therefore, the values of these parameters and the materials used in building the simulation environment for the institute are shown in Table 2.

$$
\begin{gathered}
\sigma=c * f^{(d)} \\
\varepsilon=a * f^{(b)}
\end{gathered}
$$

Where $a, b, c$, and $d$ are constants characterizing each material included in the construction of the simulation environment, which has been fixed by ITU's organization and $f$ is the frequency of the carrier band.

\begin{tabular}{|c|c|c|c|c|c|c|c|c|c|c|c|c|}
\hline \multirow{2}{*}{ Materials } & \multicolumn{2}{|c|}{$\begin{array}{c}\text { C-Band } \\
(2.4 \mathrm{GHz}) \\
\end{array}$} & \multicolumn{2}{|c|}{$\begin{array}{l}\text { S-band } \\
\text { (5 GHz) }\end{array}$} & \multicolumn{2}{|c|}{$\begin{array}{c}\text { S-band } \\
(10 \mathrm{GHz})\end{array}$} & \multicolumn{2}{|c|}{$\begin{array}{r}\text { Ku-Band } \\
(15 \mathrm{GHz})\end{array}$} & \multicolumn{2}{|c|}{$\begin{array}{c}\text { Ka-Band } \\
(28 \mathrm{GHz})\end{array}$} & \multicolumn{2}{|c|}{$\begin{array}{c}\text { MmWave } \\
\text { (39 GHz) }\end{array}$} \\
\hline & $\sigma$ & $\varepsilon$ & $\sigma$ & $\varepsilon$ & $\sigma$ & $\varepsilon$ & $\sigma$ & $\varepsilon$ & $\sigma$ & $\varepsilon$ & $\sigma$ & $\varepsilon$ \\
\hline Brick & 0.038 & 3.75 & 0.038 & 3.75 & 0.038 & 3.75 & 0.038 & 3.75 & 0.038 & 3.75 & 0.038 & 3.75 \\
\hline Concrete & 0.066 & 5.31 & 0.119 & 7 & 0.210 & 5.31 & 0.291 & 5.31 & 0.483 & 5.31 & 0.632 & 5.31 \\
\hline Metal & $10^{7}$ & 1 & $10^{7}$ & 1 & $10^{7}$ & 1 & $10^{7}$ & 1 & $10^{7}$ & 1 & $10^{7}$ & 1 \\
\hline Dense Foliage & 0.1 & 1 & 0.1 & 1 & 0.1 & 1 & 0.1 & 1 & 0.1 & 1 & 0.1 & 1 \\
\hline Wood & 0.012 & 1.99 & 0.026 & 1.99 & 0.055 & 1.99 & 0.085 & 1.99 & 0.167 & 1.99 & 0.238 & 1.99 \\
\hline Glass & 0.012 & 6.27 & 0.066 & 6.27 & 0.077 & 6.27 & 0.079 & 6.27 & 0.088 & 6.27 & 0.098 & 6.27 \\
\hline
\end{tabular}

Table 2. The conductivity $(\sigma)$ and permittivity $(\varepsilon)$ for various frequencies and materials.

The transmitting devices used and installed in an institute environment are shown in Figure 4, while the properties of each device are listed in Table 3. Two types of transmitting devices are used, the first type is a Directional transmitting device, while the second is Omni-Directional. The aim using two types of transmitting devices to identify and know which is better (Directional or Omni-Directional) to coverage the simulation institute environment. 


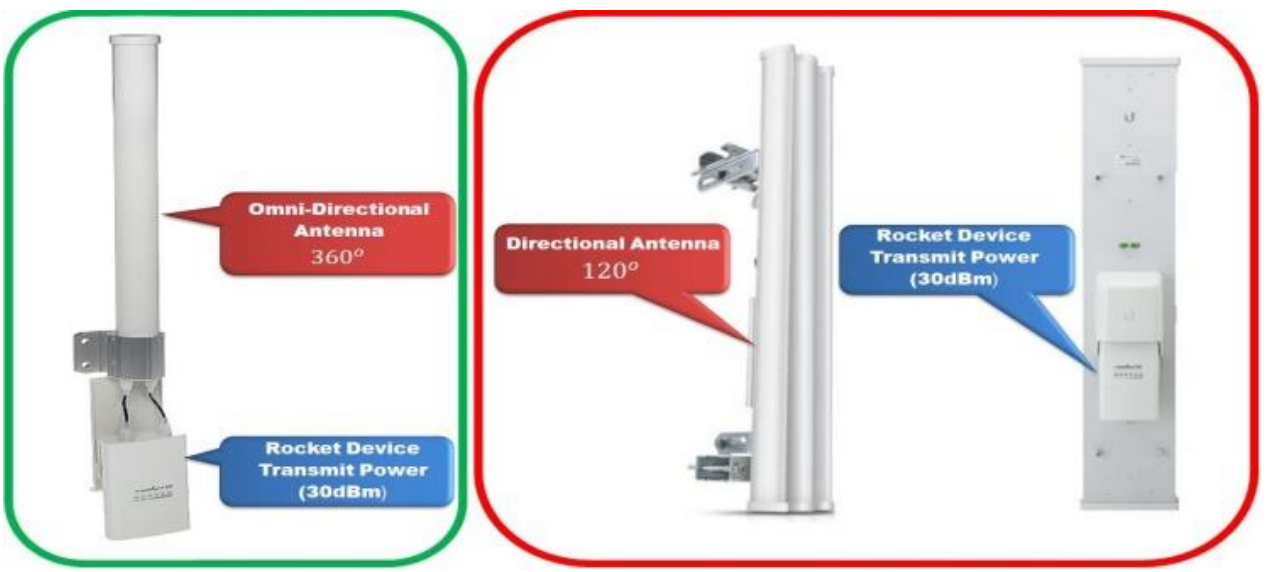

Figure 4. The transmitting devices (Directional and Omni-Directional) are installed in the institute's environment.

Table 3. Detailed properties of transmitting devices and receiving points deployed in the institute's environment.

\begin{tabular}{cccc}
\hline \multirow{2}{*}{ Properties } & Transmitting Device 1 & Transmitting Device 2 & Received Points \\
\cline { 2 - 4 } & Directional & Omni-Directional & Omni-Directional \\
\hline Polarization & Vertical & Vertical & Vertical \\
$\begin{array}{c}\text { Transmit Power } \\
(\mathbf{d B m})\end{array}$ & 30 & 30 & -- \\
$\begin{array}{c}\text { Electric Field } \\
\text { Plane Beam Width } \\
\text { Operating }\end{array}$ & $120^{\circ}$ & $360^{\circ}$ & $360^{\circ}$ \\
$\begin{array}{c}\text { Frequencies } \\
\text { Bandwidths }\end{array}$ & $2.4,5,10,15,28$, and $39 \mathrm{GHz}$ & $2.4,5,10,15,28$, and $39 \mathrm{GHz}$ & -- \\
Antenna Gain & $20,40,80,120,1300,1400 \mathrm{MHz}$ & $20,40,80,120,1300,1400 \mathrm{MHz}$ & -- \\
\hline
\end{tabular}

The total number of receiving points that were deployed in the institute's environment is 436 points, so 286 receiving points were deployed on the Ground Floor as shown in Figure 5 and 75 receiving points on the First Floor as shown in Figure 6(a) and 75 receiving points on the Second Floor as shown in Figure 6(b). In addition, the distance between one receiving point and another that was equal is $4 \mathrm{~m}$. While the heights of all these points for Ground, First, and Second are 1.20, 4.7, and $8.2 \mathrm{~m}$ respectively, in terms of a person has a smartphone or laptop in hand and receiving signals from the transmitting devices.

Moreover, the height of the transmitting devices is $2.5 \mathrm{~m}$ for both (Directional and Omni-Directional), so that the Omni-Directional transmitting device was installed in the middle of the institute's environment because the signal transmitted from this device is sent in all directions as shown in Figure 5. While the Directional transmitting device was installed at the beginning of the entrance to the institute is because the directional device transmits its signal in one direction, as shown in Figure 5 and Figure 6.

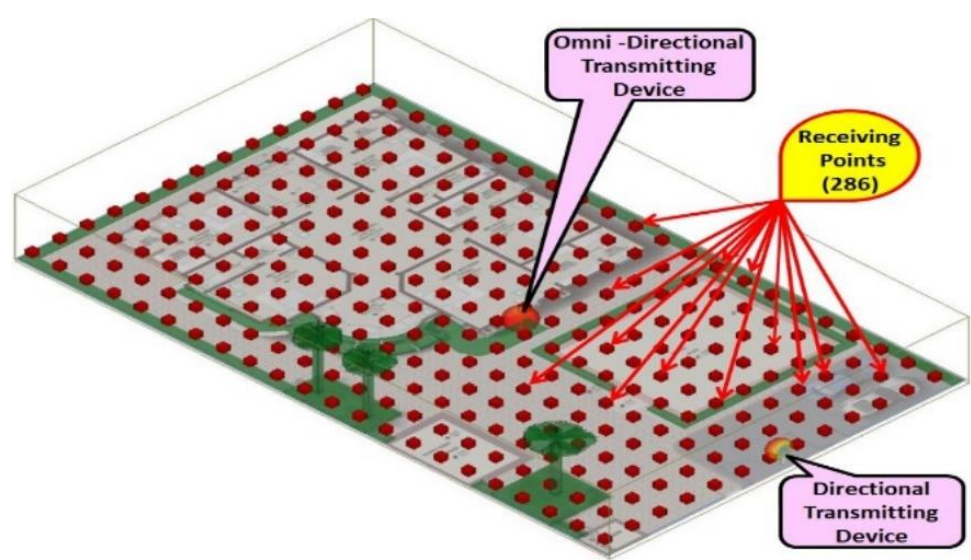

Figure 5. The transmitting devices (Directional and Omni-Directional) and receiving points are deployed on the Ground Floor. 


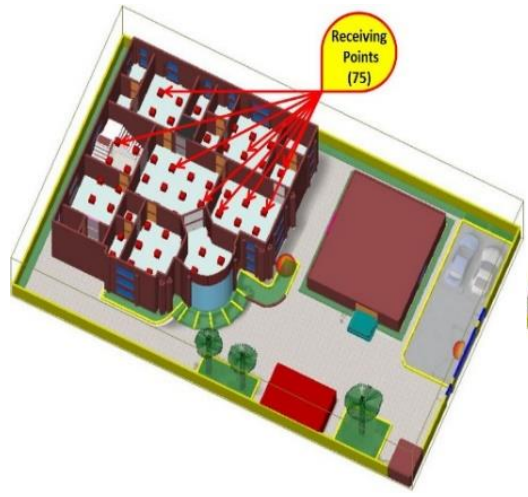

(a)

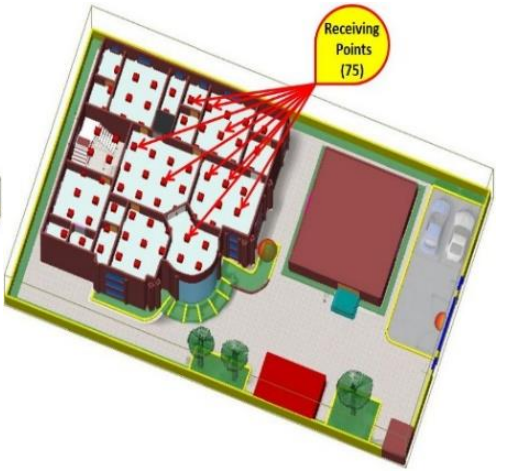

(b)

Figure 6. The receiving points deployed in the institute's environment for (a) First Floor and (b) Second Floor.

\section{RESULTS AND DISCUSSION}

In this section, all the results that were relied upon in this paper will be analyzed and discussed. The most important parameters were relied on to know the performance of the wireless network proposed in this paper, and these parameters are the transmission strength of the transmitting devices (Directional and OmniDirectional), path losses, path gain, RSS. While in the final part of this section, a comparison between the antennas (Directional and Omni-Directional) will be presented by knowing which antenna is better to cover the institute's environment so that the comparison is based on the CR and the RSQR.

\subsection{Path Losses $\left(L_{\text {Path }}\right)$}

The path losses in $(\mathrm{dB})$ for each transmitting device (Directional and Omni-Directional) installed in the institute environment at frequency $2.4 \mathrm{GHz}$ are illustrated in Figure 7 . We note that there is an inverse relationship between path losses and distance so that as the distance increases, the path losses will increase and vice versa. In addition, we note that the losses facing the paths in the Omni-Directional antenna are less than the losses facing the paths in the Directional antenna, because the Directional antenna directs its power and signal in one direction, so the signals will collide with barriers, obstacles, and walls. Therefore, walls and obstacles will reflect, break, absorb or scatter the signal, as the signals will reach weak receiving points with high losses.

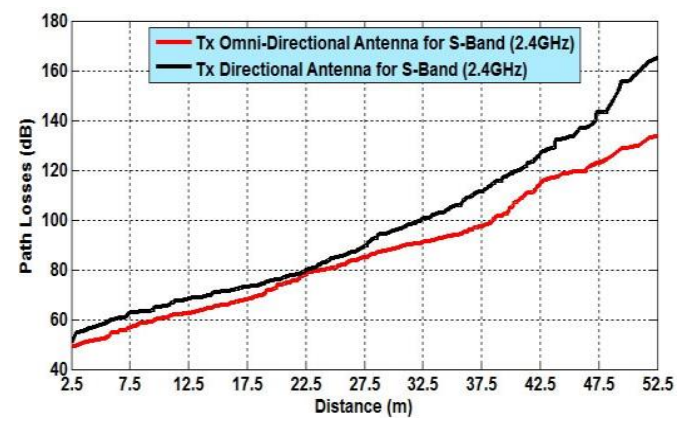

Figure 7. The path losses in $(\mathrm{dB})$ versus distance at $2.4 \mathrm{GHz}$ for transmitting devices (Directional and Omni-

Directional).

Moreover, when comparing the path losses at different and multi-band frequencies that are S-Band $(2.4 \mathrm{GHz}), \mathrm{C}-\mathrm{Band}(5 \mathrm{GHz}), \mathrm{C}-\mathrm{Band}(10 \mathrm{GHz}), \mathrm{Ku}-\mathrm{Band}(15 \mathrm{GHz}), \mathrm{Ka}-\mathrm{Band}(28 \mathrm{GHz})$, and MmWave (39GHz) for the Directional transmitting devices are shown in Figure 8(a), and for the Omni-Directional transmitting devices are shown in Figure 8(b). From the information shown in the two Figures, we note that there is an inverse relationship between path losses and frequency so that as the frequency increases, path losses will increase. We also notice that the path losses at MmWave $(39 \mathrm{GHz})$ are much higher than the path losses at $\mathrm{S}$ Band $(2.4 \mathrm{GHz})$ because as the frequency increases, the wavelength will decrease. For this, the signal did not reach very far distances and also did not penetrate a greater number of barriers and obstacles, so this will lead to an increase in path losses. Likewise, some of the signals did not reach the receiving points at all due to a large number of collisions. 


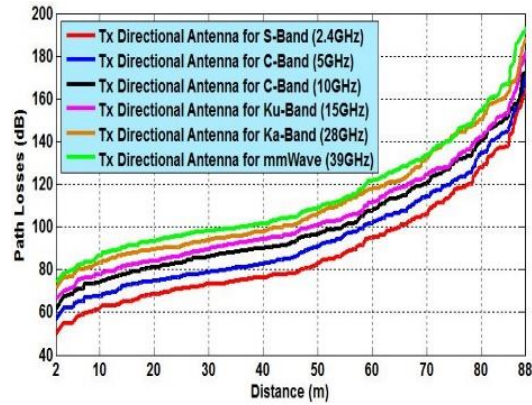

(a)

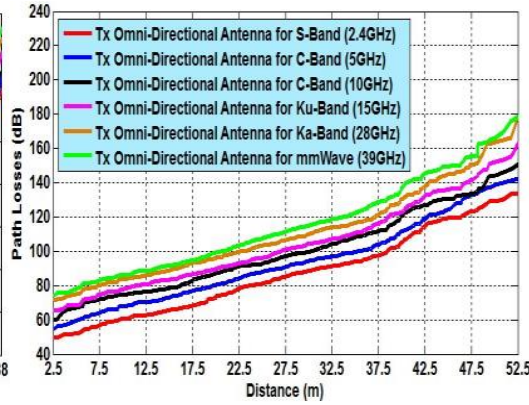

(b)

Figure 8. Path losses at multi-bands frequencies that are S-Band (2.4GHz), C-Band (5GHz), C-Band (10GHz), Ku-Band (15GHz), Ka-Band (28GHz), and MmWave (39GHz).

\subsection{Path Gain $\left(\mathbf{G}_{\text {Path }}\right)$}

The path gain of two antennas (Directional and Omni-Directional) at C-Band (5GHz) is shown in Figure 9. It is observed that the path gain decreases gradually when the distance between the transmitting and receiving antennas increases. This indicates that the relationship between them is an inverse relationship. In addition, it was also observed that the gain of the Omni-Directional antenna is much better than the Directional antenna because the Omni-Directional antenna transmits its power in all directions, so it is not affected by collisions and obstacles much.

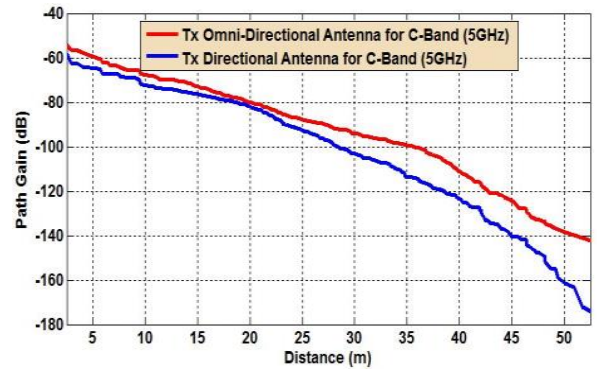

Figure 9. Path gain versus various distances for two antennas (Directional and Omni-Directional) at C-band $(5 \mathrm{GHz})$.

Furthermore, the path gain also decreases when the frequency increases, as shown in Figure 10(a, b), because the transmitting devices that have high frequencies, these devices have a very short coverage range, so we notice that the path gain at $\mathrm{S}-\mathrm{Band}(2.4 \mathrm{GHz})$ is much better than MmWave $(39 \mathrm{GHz})$.

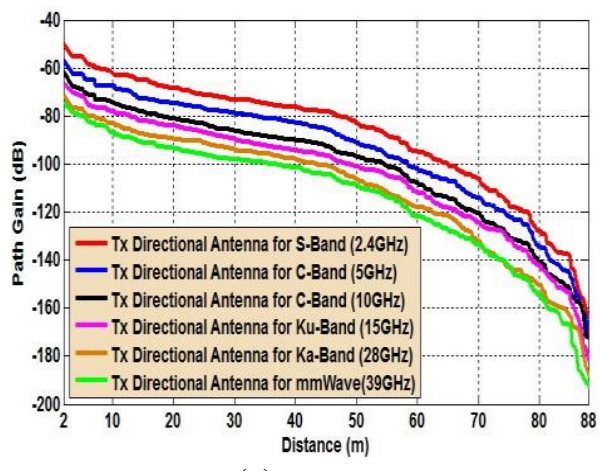

(a)

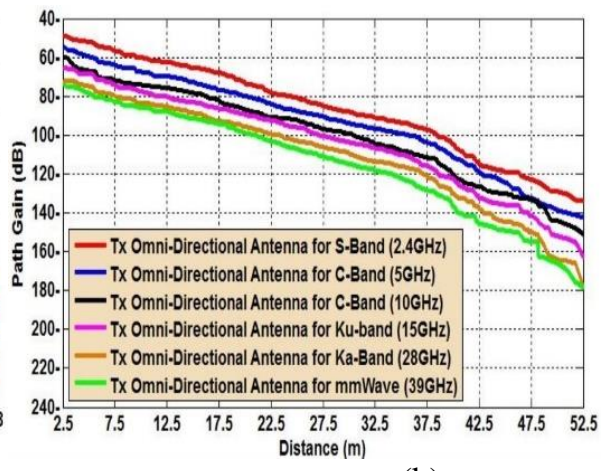

(b)

Figure 10. The path gain for the two antennas (Directional and Omni-Directional) at the multi-band's frequencies are S-Band (2.4GHz), C-Band (5GHz), C-Band (10GHz), Ku-Band (15GHz), Ka-Band (28GHz), and MmWave $(39 \mathrm{GHz})$.

\subsection{Received Signal Strength (RSS)}

The values of the RSS for the Directional transmitting device and the Omni-Directional transmitting device at the multi-bands frequencies as S-Band (2.4GHz), C-Band (5GHz), C-Band (10GHz), Ku-Band 
$(15 \mathrm{GHz}), \mathrm{Ka}-\mathrm{Band}(28 \mathrm{GHz})$, and MmWave $(39 \mathrm{GHz})$ are shown in Figure 11. Through the values shown in Figure 11, we notice that the received signal strength gradually decreases with increasing the frequency, as the signal strength value at the frequency of S-Band $(2.4 \mathrm{GHz})$ ranges between 1.73 and $-83.17 \mathrm{dBm}$ which is much better than the signal strength values at the frequency of MmWave $(39 \mathrm{GHz})$, which ranges between -23.02 and $-163.3 \mathrm{dBm}$. It was also noted that the signal strength at the Omni-Directional antenna is much better than the Directional antenna because there are many effects that prevent, obstruct, reflect and disperse the paths of the signals transmitted to the receiving points, and the most important of these effects are the materials used in building walls, barriers, and others. In addition, it was noted that the concrete material reflects the signal path. The bricks metal, glass, and wood all these materials disperse the signal strength transmitted from the transmitter to the receiving points.

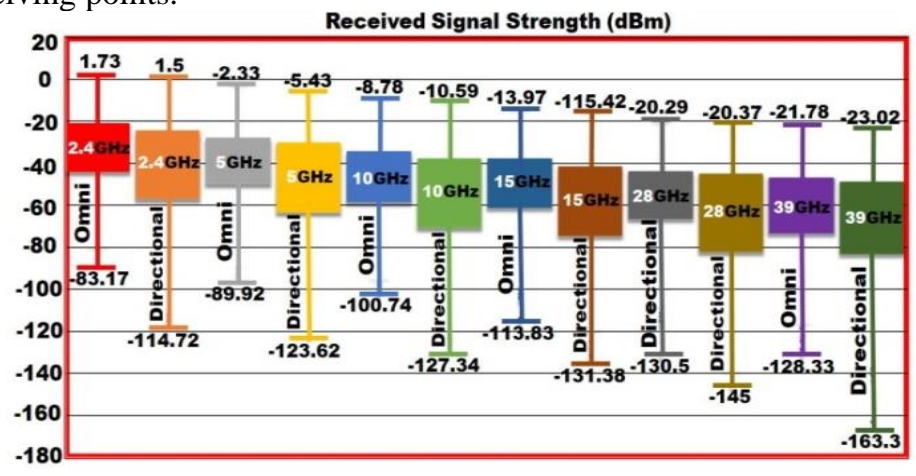

Figure 11. The RSS of a Directional and Omni-Directional antenna at multi-band frequencies that are S-Band $(2.4 \mathrm{GHz}), \mathrm{C}-\mathrm{Band}(5 \mathrm{GHz}), \mathrm{C}-\mathrm{Band}(10 \mathrm{GHz}), \mathrm{Ku}-\mathrm{Band}(15 \mathrm{GHz}), \mathrm{Ka}-\mathrm{Band}(28 \mathrm{GHz})$, and MmWave (39GHz).

\subsection{Strongest Received Power}

The signal path when transmitted from the transmitting device antenna to the receiving antennas will pass through many obstacles and barriers, especially when the receiving antennas are in NLOS regions. These barriers and obstacles that impede the signals path are made of different materials and each material has a different effect, so that it has been observed that concrete reflects or breaks the signal path, while the bricks disperse and some of them absorb the ability of the signal path, and this dispersion and absorption depends on the thickness of the bricks. In addition, wood, glass, dense foliage, and metal have less effect on the signal path than concrete and bricks.

The received signal power strength for all receiving points deployed over the institute's environment and for all floors (Ground, First, and Second) at the frequency of $2.4 \mathrm{GHz}$ are shown in Figure $12(\mathrm{a}, \mathrm{b})$. When focusing carefully on Figure 12(a) and Figure 12(b), we notice that the emission of an Omni-Directional antenna is much better than a Directional antenna because the Omni-Directional antenna transmits its power at $360^{\circ}$. Therefore, the path power when hitting an obstacle will generate attenuation and is less noise than the maximum power of the path when transmitted from the Directional antenna. In addition, the Directional antenna used in this paper directs its power at $120^{\circ}$ and in one direction, so when the maximum power of the path collides with a barrier, it will generate severe attenuation, and for this, the power will reach the receiving points in the NLOS regions that are very weak and some of them did not reach because of a large number of barriers. Moreover, we notice that there are many colors in the two Figures, and these colors represent the strength of the power that the user receives, so the red color represents the strongest power that reaches the user, the yellow color represents medium power, the green color represents a weak power, and the blue color represents a very weak power that will reach the users and some of them it did not finally reach its extreme weakness.

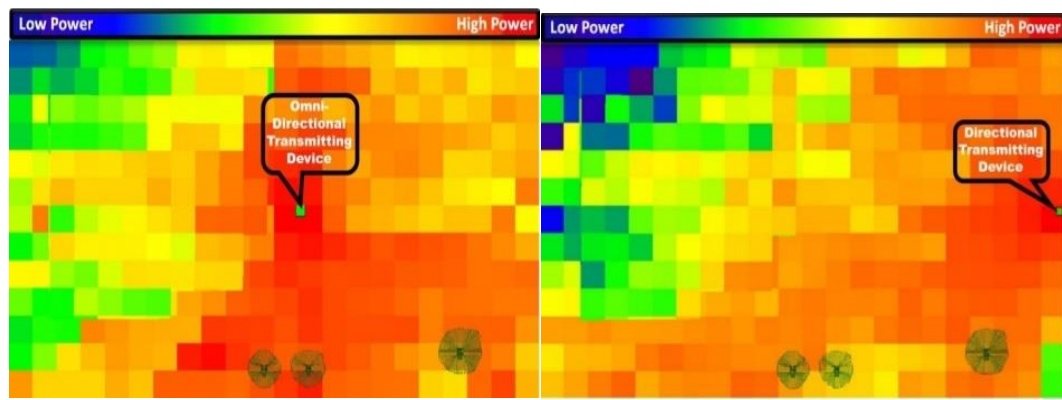

(a)

(b)

Figure 12. The strongest received power by the users at S-Band (2.4GHz) for (a) Omni-Directional transmitting device and (b) Directional transmitting device. 


\subsection{Transmitting Device Antennas Performance Comparison}

The performance comparison of transmitting devices for two antennas (Directional and OmniDirectional) at different frequency bands are S-Band $(2.4 \mathrm{GHz}), \mathrm{C}-\mathrm{B}$ and $(5 \mathrm{GHz}), \mathrm{C}-\mathrm{Band}(10 \mathrm{GHz}), \mathrm{Ku}-\mathrm{Band}$ $(15 \mathrm{GHz}), \mathrm{Ka}-\mathrm{Band}(28 \mathrm{GHz})$, and MmWave $(39 \mathrm{GHz})$ shown in Figure 13. The comparison depended on the most important parameters to determine the extent of the transmitter's ability to deliver signals for the receiving points so that the focus was on two main parameters to know the duration of stability and wireless network coverage designed in this paper. The first parameter is to calculate the CR of the institute environment in an integrated manner and for various frequencies. The second parameter is the calculation of the RSQR that reaches each user. The comparison and all calculations were programmed using Matlab Software, as well as designing a Graphical User Interface (GUI) in order to view and monitor parameters calculations for various bands. Based on the results obtained and shown in Figure 13, we noticed that the CR of the Omni-Directional antenna is $83.2184 \%, 77.2414 \%, 60.6897 \%, 51.9540 \%, 38.1609 \%$, and $31.0345 \%$ for frequencies $2.4,5,10$, 15,28 , and $39 \mathrm{GHz}$ respectively, which are much better than the CR for a directional antenna which is $66.6667 \%, 55.1724 \%, 49.1954 \%, 39.0805 \%, 32.8736 \%$, and $27.1264 \%$ for frequencies $2.4,5,10,15,28$, and $39 \mathrm{GHz}$ respectively when the RSS selected is $\geq(-50 \mathrm{dBm})$. In addition, the RSQR of the Omni-Directional antenna is quite good compared to the Directional antenna. There are several reasons that led to the Directional antenna being worse than the Omni-Directional antenna, the most important of which are the transmission direction power, the number of barriers, obstacles, and the different building materials. All of them made the Directional antenna unable to work well to transmit signals from outdoor to indoor environments. Finally, we note that the wireless network coverage and the signal quality transmit from the transmitting device to the receiving points and for the two antennas (Directional and Omni-Directional) at S-Band (2.4 GHz) is much better than other bands.

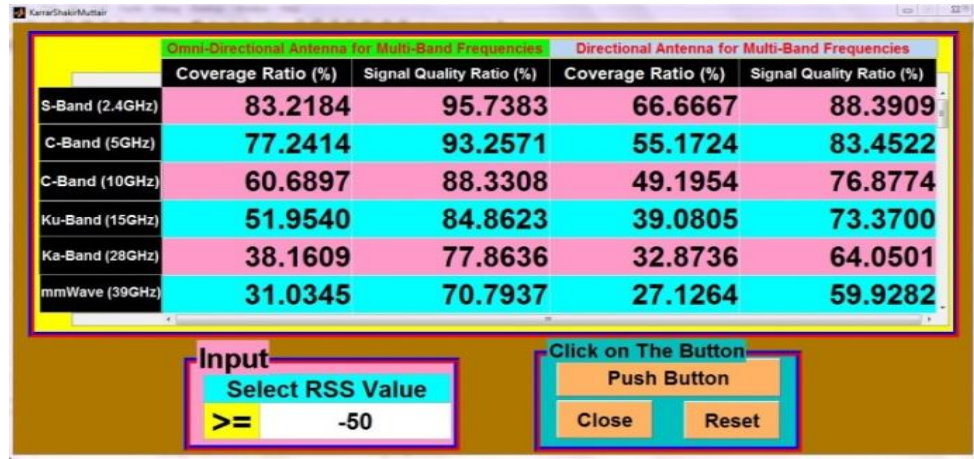

Figure 13. The coverage ratio and signal quality for the two antennas (Directional and Omni-Directional) at different bands in the Matlab on the GUI.

\section{CONCLUSION}

This paper examines the ideal coverage of the target institute's environment. In this work, standards and basic parameters are used in order to measure the performance level of the designed network and its ability to cover the institute environment. The most important of these parameters are CR, RSQR, $\mathrm{L}_{\text {Path }}, \mathrm{G}_{\mathrm{Path}}$, RSS, and the strongest receiving power for all the receiving points deployed in the institute's environment. Based on the results obtained from the WIP and the Matlab Software, we have concluded three main reasons. In the first conclusion, we noticed that the directional antennas generate high attenuation and noise compared to the OmniDirectional antennas, especially in the NLOS regions because the Directional antennas direct their power in one direction, and whenever the power is directed in one direction, it will collide with many barriers and obstacles, so the attenuation of the signal path will increase. In addition, the CR of the Omni-Directional antenna is $83.2184 \%$ at $\mathrm{S}-\mathrm{B}$ and $(2.4 \mathrm{GHz})$ and this ratio is good compared to the CR of the Directional antenna, which is $66.6667 \%$ for the same frequency, gain, and transmit power. In the second conclusion, we noticed that the materials used in building the institute's environment have a great effect on obstructing the direction of the signal path transmitted from the transmitting devices to the receiving points so that we noticed that the concrete material reflects and breaks the signal path, and the materials of brick, wood, metal, glass, dense foliage, some of which are dispersed and others it absorbs signal strength. In the last conclusion, we noticed that as the band frequency increases, it will lead to the deterioration of the wireless network, as we note that CR and RSQR at S-Band $(2.4 \mathrm{GHz})$ are $83.2184 \%$ and $95.7383 \%$, which are much better than MmWave $(39 \mathrm{GHz})$, which are $31.0345 \%$ and $70.7937 \%$. The main reason behind this degradation is that the high band frequency has a short wavelength, so it is difficult to penetrate a large number of obstacles, so the network will degrade. In future work, we will propose a new scenario in order to present a wireless sensor network with the 
least number of sensors to monitor patients' status remotely. Also, linking this network with 5G technologies to keep pace with the development in medical wireless systems.

\section{ACKNOWLEDGEMENTS}

We extend our sincere thanks and gratitude to the Islamic University (https://iunajaf.edu.iq/) and the Middle Technical University for the continuous support they provided to us, which is the main gem for accomplishing this academic work that simulates the development in the $5 \mathrm{G}$ wireless communications.

\section{REFERENCES}

[1] K. Pahlavan and P. Krishnamurthy, "Evolution and Impact of Wi-Fi Technology and Applications: A Historical Perspective," International Journal of Wireless Information Networks, no. 28, pp. 3-19, 2020.

[2] A. Zanella, et al., "Internet of things for smart cities," IEEE Internet of Things journal, vol. 1, no. 1, pp. 22-32, 2014.

[3] P. Dong, et al., "Enhancing vehicular communication using 5g-enabled smart collaborative networking," IEEE Wireless Communications, vol. 24, no. 6, pp. 72-79, 2017.

[4] M. H. Seko and F. S. Correra, "Penta-band antenna for global system for mobile communications/digital cellular system/personal communications service/universal mobile telecommunications system/wireless local area network operation in portable devices," IET Microw. Antennas Propag., vol. 13, no. 7, pp. 930-935, 2019.

[5] Karrar Shakir Muttair, et al., "A New Design of mm-Wave MIMO Antenna with High Isolation for 5G Applications," International Journal of Microwave and Optical Technology, vol. 16, no. 4, pp. 370-379, 2021.

[6] S. Hossain, "5G Wireless Communication Systems," American Journal of Engineering Research (AJER), vol. 02, no. 10 , pp. 344-353, 2013.

[7] Karrar S. Muttair, et al., "Outdoor to Indoor Wireless Propagation Simulation Model for 5G Band Frequencies," The Fourth Postgraduate Engineering Conference, IOP Publishing, vol. 745, no. 1, pp. 012034, 2020.

[8] Sun-Kuk Noh, and D. You Choi, "Propagation Model in Indoor and Outdoor for the LTE Communications," International Journal of Antennas and Propagation, vol. 2019, no. 3134613, pp. 1-6, 2019.

[9] N. Ullah, and A. Tekin, "Omni-Directional Wideband Antenna Array with Solar Cells," Journal of Microwaves, Optoelectronics and Electromagnetic Applications, vol. 20, no. 1, pp. 60-74, 2021.

[10] H. Ning Dai, et al., "An overview of using directional antennas in wireless networks," International Journal of Communication Systems, vol. 26, pp. 413-448, 2013.

[11] Y. Wang, et al., "Directional neighbor discovery in mmWave wireless networks," Digital Communications and Networks, vol. 7, no. 1, pp. 1-15, 2021.

[12] A. Dhekne, M. Gowda, and R. R. Choudhury, "Extending cell tower coverage through drones," in Proceedings of the $18^{\text {th }}$ International Workshop on Mobile Computing Systems and Applications. ACM, pp. 7-12, 2017.

[13] M. J. C. Jimenez, K. Arana, and M. R. Arias, "Outdoor-to-indoor propagation mechanisms in multistorey building for $0.85 \mathrm{ghz}$ and $1.9 \mathrm{ghz}$ bands," IEEE $37^{\text {th }}$ Central America and Panama Convention (CONCAPAN XXXVII), pp. $1-6,2017$.

[14] H. Kunsei, "Improving wireless communications in underground mines using reconfigurable antennas," University of Queensland, Australia, 2018.

[15] Karrar S. Muttair, et al., "Performance Comparison of Multi-band Frequencies for Outdoor Communication," Applied Computing to Support Industry: Innovation and Technology. Communications in Computer and Information Science (Springer), vol. 1174, pp. 476-487, 2020.

[16] G. R. MacCartney, et al., "Path loss models for $5 \mathrm{~g}$ millimeter wave propagation channels in urban microcells," IEEE Globecom., pp. 3948-3953, 2013.

[17] S. State College Remcom Inc., 315 S. Allen St., "Wireless insite reference manual," IEEE Transactions on Vehicular Technology, vol. 58, no. 1, pp. 16801, 2012.

[18] O. A. S. Al-Ani, Karrar S. Muttair, and M. F. Mosleh, "Outdoor transmitter localization using multiscale algorithm," International Journal of Simulation Systems, Science \& Technology (United Kingdom), pp. 3.1-3.7, 2019.

[19] S. I. Popoola, et al., "Radio Access Technologies for Sustainable Deployment of 5G Networks in Emerging Markets," International Journal of Applied Engineering Research, vol. 12, no. 24, pp. 14154-14172, 2017.

[20] A. A. A. Maitra, "Studies on the Interrelation of Ku-band scintillations and rain attenuation over an Earth-Space Path on the basis of Their Static and Dynamic Spectral Analysis," Journal of Atmospheric Solar Terrestrial Physics, vol. 73, pp. 516-527, 2011.

[21] D. Martinez-de-Rioja, et al., "Multibeam Reflectarrays in Ka-Band for Efficient Antenna Farms Onboard Broadband Communication Satellites," Sensors, vol. 21, no. 207, pp. 1-17, 2021.

[22] Karrar S. Muttair, et al., "Outdoor Millimeter-Wave Propagation Simulation Model for 5G Band Frequencies," $2^{\text {nd }}$ International Conference on Electrical, Communication, Computer, Power and Control Engineering (IEEE), pp. 41-46, 2019.

[23] Y. Niu, Y. Li, et al., "A survey of millimeter wave communications (mmwave) for 5g: opportunities and challenges," Wireless Networks (Springer), vol. 21, no. 8, pp. 2657-2676, 2015. 


\section{BIOGRAPHY OF AUTHORS}
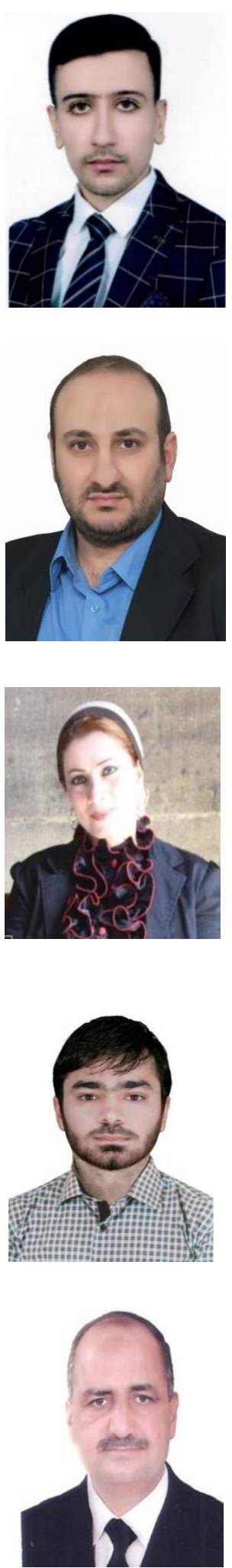

Karrar Shakir Muttair: received a B.Sc. degree from the Islamic University/ Iraq in 2016 in the field of Computer Techniques Engineering / Computer Communications Networks; he worked in the same University up to 2017 as a lecturer. He was awarded M.Sc. degrees from Middle Technique University / Electrical Engineering Technical College / Baghdad in 2019 in the same field of specialization. Through those periods and up to now, he worked as a lecturer in the field of Computer Communications Networks, Security of Computer \& Networks, RealTime System, and Microprocessor Architecture. He published several types of research in the field of Communications Engineering. He has been awarded several awards and certificates of thanks and appreciation in the field of his work. His research interests are Computer Techniques Engineering, Computer Communications Networks, Antennas, Indoor and Outdoor Wireless Networks.

Ali Z. Ghazi Zahid: born in Iraq, studied in Iraq until 1993, finished high school, and awarded B.Sc. degree in computer engineering from Libya graduated with honor in 2002 . Worked as a professional engineer with 7 years of experience in information technology, computer and networking industry as a network administrator, systems installation. Has proven record of project management success evaluating, installing, and supporting all areas of the network and ensuring appropriate resolution of problems during network outages, and Web development Awarded M.Sc. degree in IMEN/ UKM/ Malaysia in Micro-engineering and Nano-electronics in 2009. Worked in the Electric, Electronic, and Computer Education field. Awarded Ph.D. in Micro-Engineering and Nano-Electronics from IMEN/ UKM/ Malaysia in 2015. Currently heading the department of computer techniques engineering in college of Technical Engineering, The Islamic University in Al-Najaf.

Oras A. Al-Ani: received the B.Sc. and M.Sc. degrees in Laser and Optoelectronic Engineering from Al-Nahreen University, Iraq, in 2000 and 2002, respectively, and Ph.D. in Nanomaterialbased solar cell from Newcastle University, UK. She has officially represented Newcastle University students at the NUS National Conference (19-21 April 2016 in Brighton) as an elected self-defining woman delegate. Dr. Al-Ani is a member of IEEE, IET, and Al-Kindi Society for Engineers; and awarded several awards for the best paper and presentation. Oras served as the Event Coordinator for the Annual Research Conference at Newcastle University and she acted as the School Representative of research students at Newcastle University.

Ahmed Mohammed Q. AL-Asadi: received a B.Sc. degree from the Alkafeel University/ Iraq in 2015 in the field of Technical Engineering / Computer Technology Engineering. He was awarded M.Sc. degrees from Middle Technique University / Electrical Engineering Technical College/ Baghdad in 2019 in the same field of specialization. Through those periods and up to now, he worked in the field of Computer Communications Networks. He published several types of research in the field of Communications, Networks Engineering, and Wireless Sensor Networks.

Mahmood F. Mosleh: received a Diploma degree from the Institute of Technology/ Baghdad in 1978 in the field of electronic Technique; he worked in the same institute up to 1992 as a Technical Trainer. He was awarded B.Sc., M.Sc., and Ph.D. degrees from the University of Technology in 1996, 1999, and 2008 respectively. Through those periods and up to now, he has worked as an advanced Trainer, Assistance Lecturer, Lecturer, Assistance Professor, and Professor in the Electrical Engineering Technical College/ Baghdad. He published about 100 papers in various journals in the field of Communications Engineering. He has a patent for designing a palm climber robot. He supervised $25 \mathrm{M} . \mathrm{Sc}$. and $10 \mathrm{Ph} . \mathrm{D}$. students. Currently, he is the Chairman of the Iraqi International Electro-Technical Committee. 